Supporting Information File

\title{
Sweetness Reduces Cytotoxicity and Enables Faster Cellular Uptake of Sub-30 nm Amphiphilic Nanoparticles
}

Carlos E. de Castro, ${ }^{\dagger}$ Caroline A. S. Ribeiro,${ }^{\dagger}$ Maria C.C. da Silva,${ }^{\dagger}$ Alexandre Gonçalves Dal-Bó, ${ }^{\ddagger}$ and Fernando C. Giacomelli ${ }^{\dagger}, *$

† Centro de Ciências Naturais e Humanas, Universidade Federal do ABC, 09210-580 Santo André - SP, Brazil.

* Universidade do Extremo Sul Catarinense - UNESC, Av. Universitária 1105, 88806000, Criciúma, SC, Brazil.

*Corresponding Author: Fernando Carlos Giacomelli

e-mail. fernando.giacomelli@ufabc.edu.br 

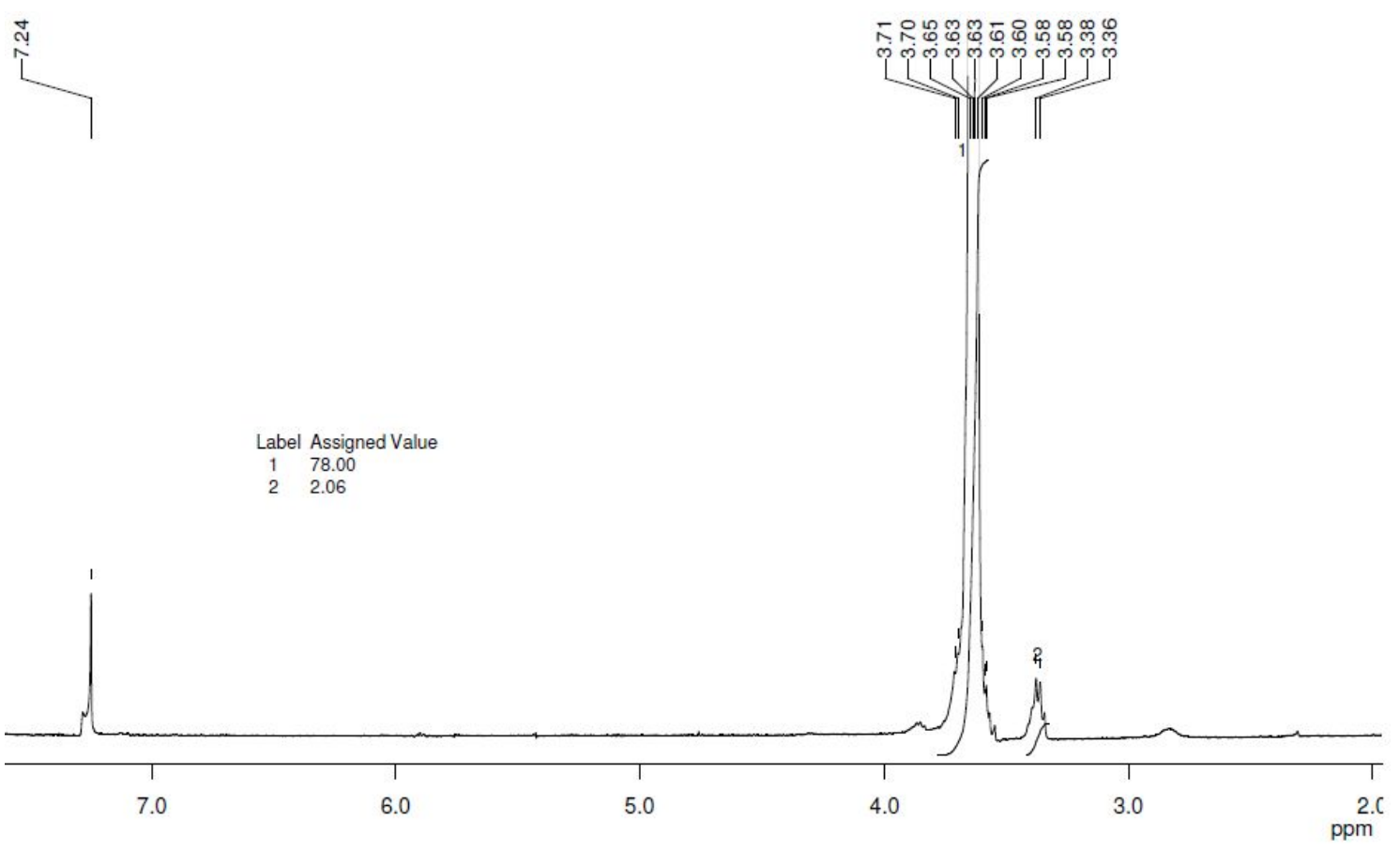

Figure S1. ${ }^{1} \mathrm{H}$ NMR spectra of mono-azide-PEO ${ }_{900}$ (1) in $\mathrm{CDCl}_{3}$.

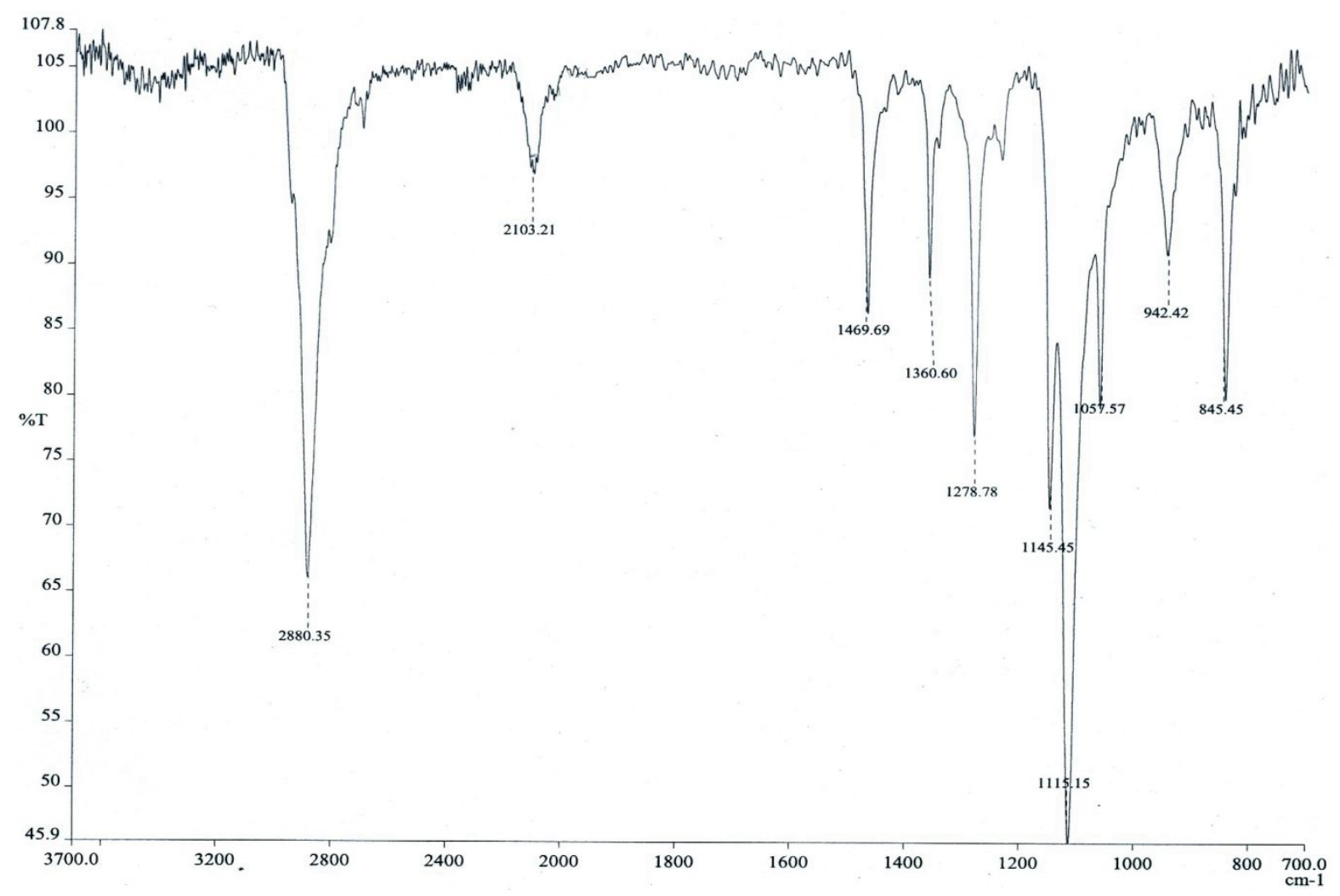

Figure S2. FTIR spectra of mono-azide- $\mathrm{PEO}_{900}$ (1). 


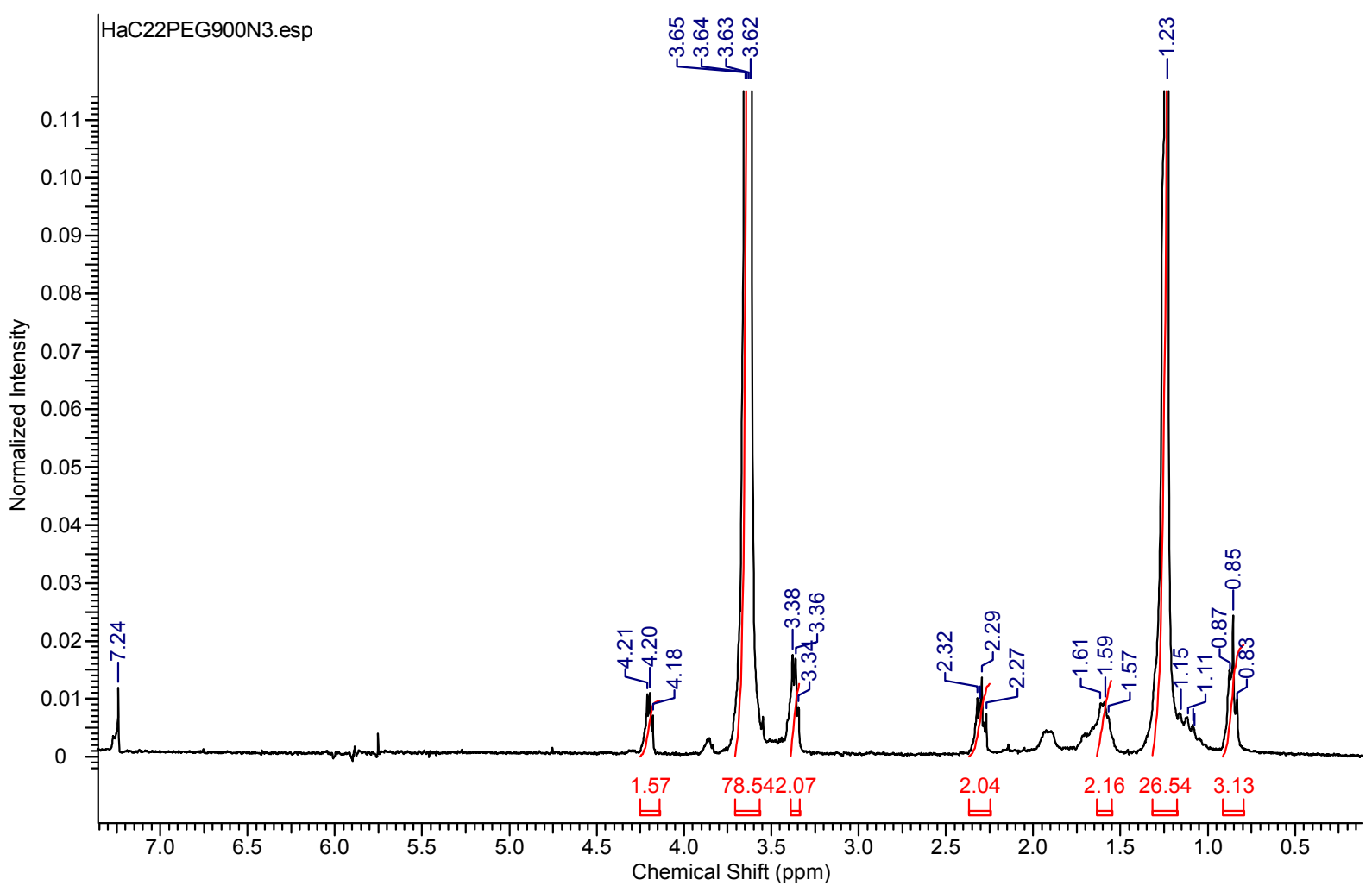

Figure S3. ${ }^{1} \mathrm{H}$ NMR spectra of azide- $\mathrm{PEO}_{900}$-docosanate (2) in $\mathrm{CDCl}_{3}$.

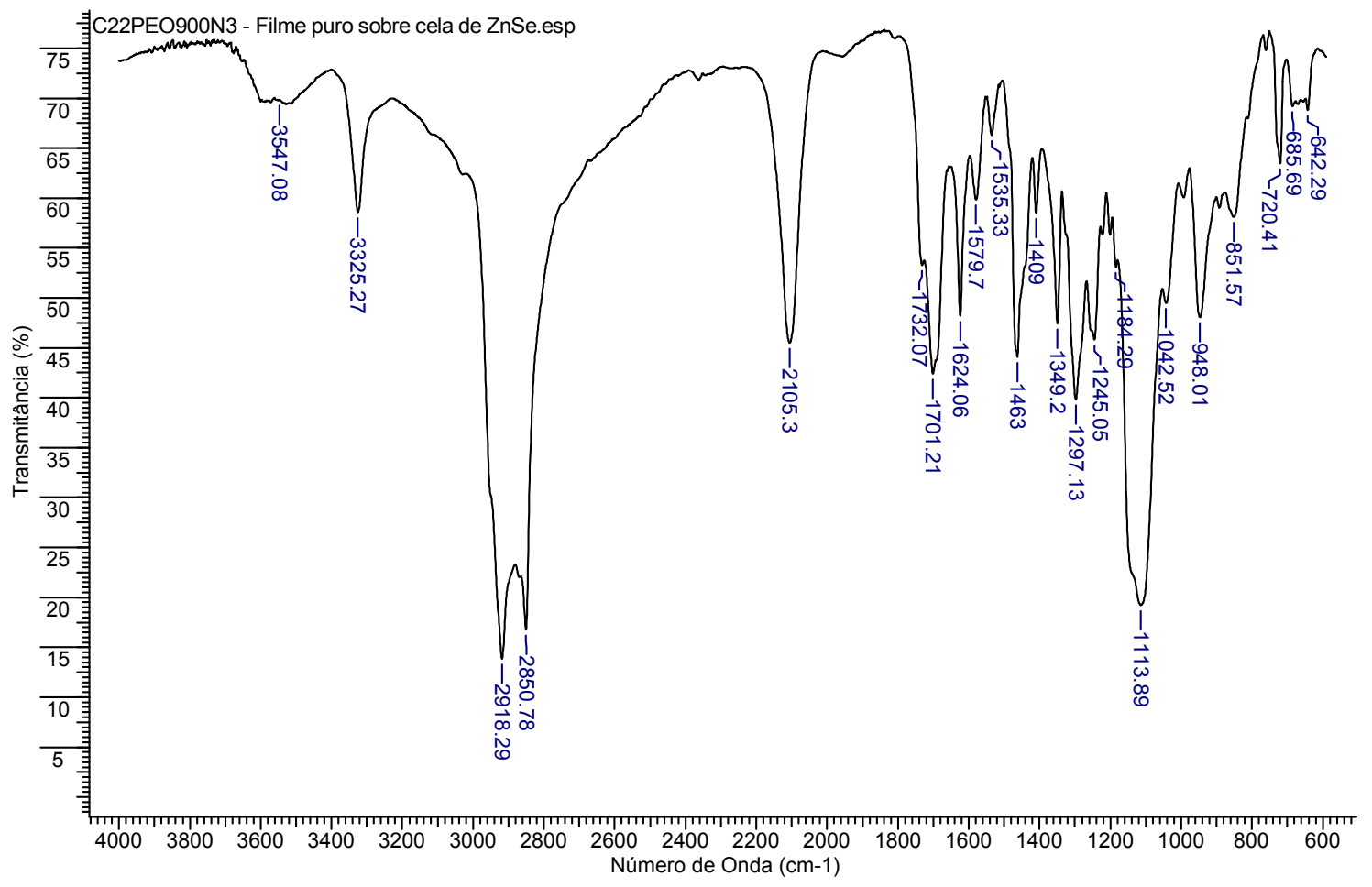

Figure S4. FTIR spectra of azide-PEO ${ }_{900}$-docosanate (2). 


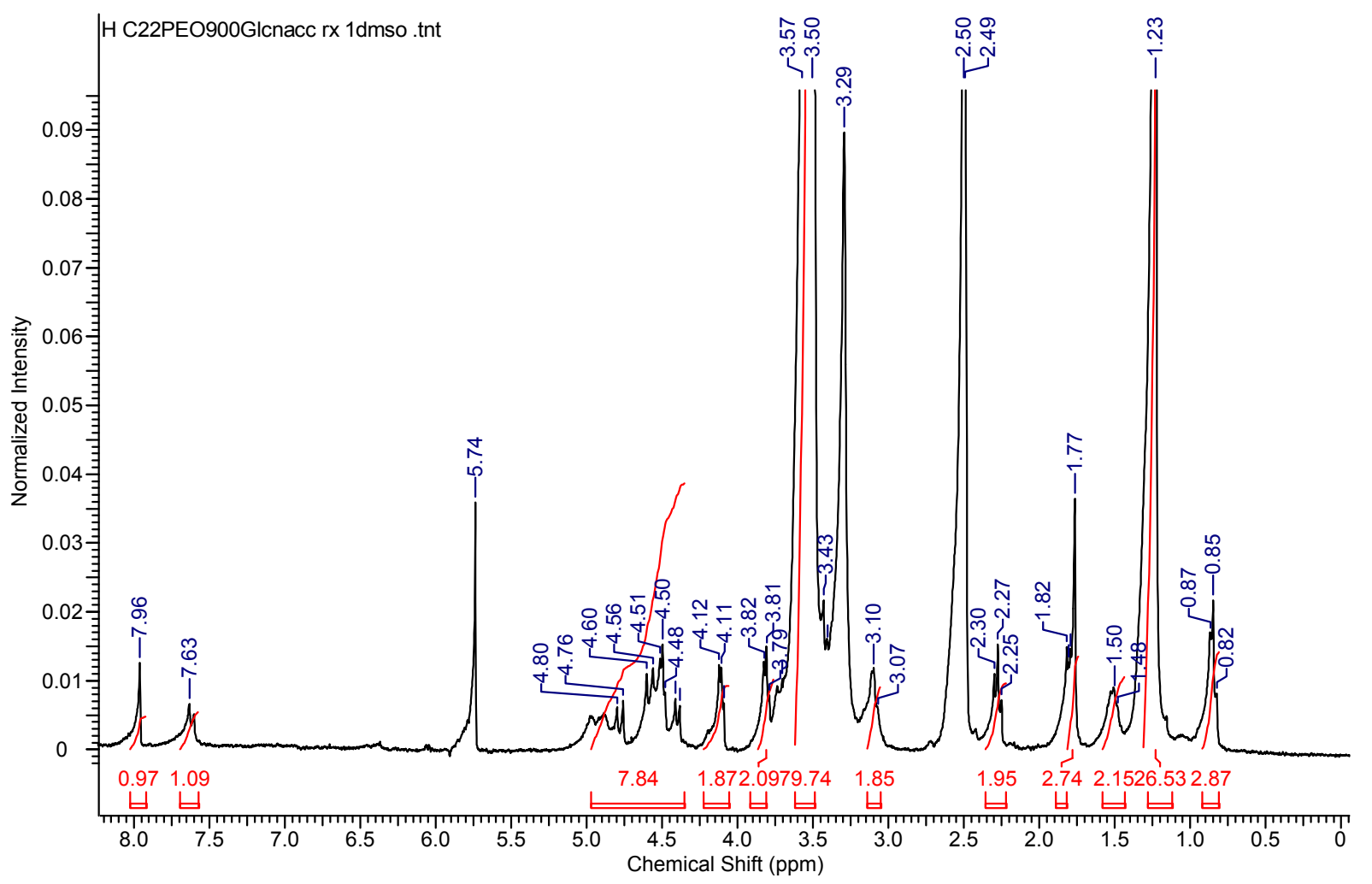

Figure S5. ${ }^{1} \mathrm{H}$ NMR spectra of $\mathrm{C}_{22} \mathrm{PEO}_{900}$ GlcNAc (3) in DMSO- $d_{6}$.

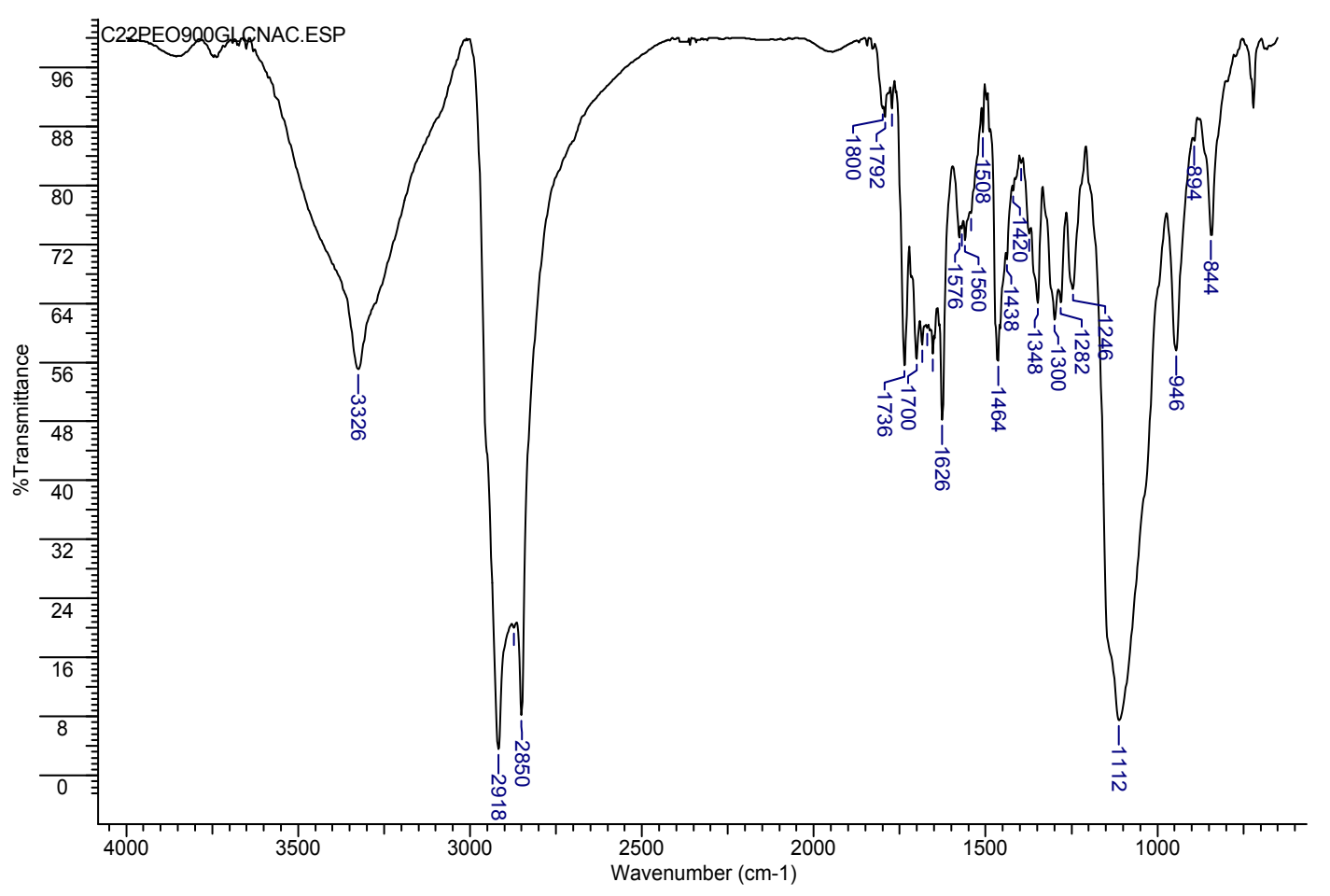

Figure S6. FTIR spectra of $\mathrm{C}_{22} \mathrm{PEO}_{900} \mathrm{GlcNAc}(3)$. 


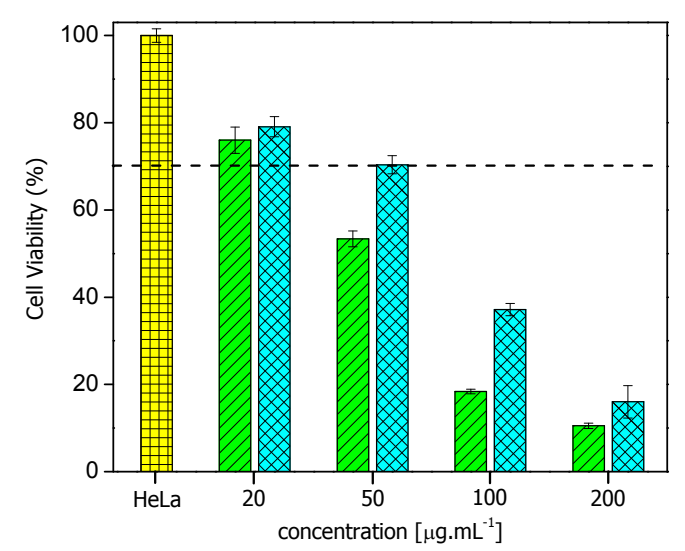

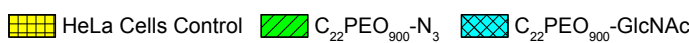

Figure S7. Viability of $\mathrm{HeLa}$ cells treated with varying concentrations of $\mathrm{C}_{22} \mathrm{PEO}_{900}-\mathrm{N}_{3}$ and $\mathrm{C}_{22} \mathrm{PEO}_{900}$-GlcNAc nanoparticles after $24 \mathrm{~h}$ incubation time.

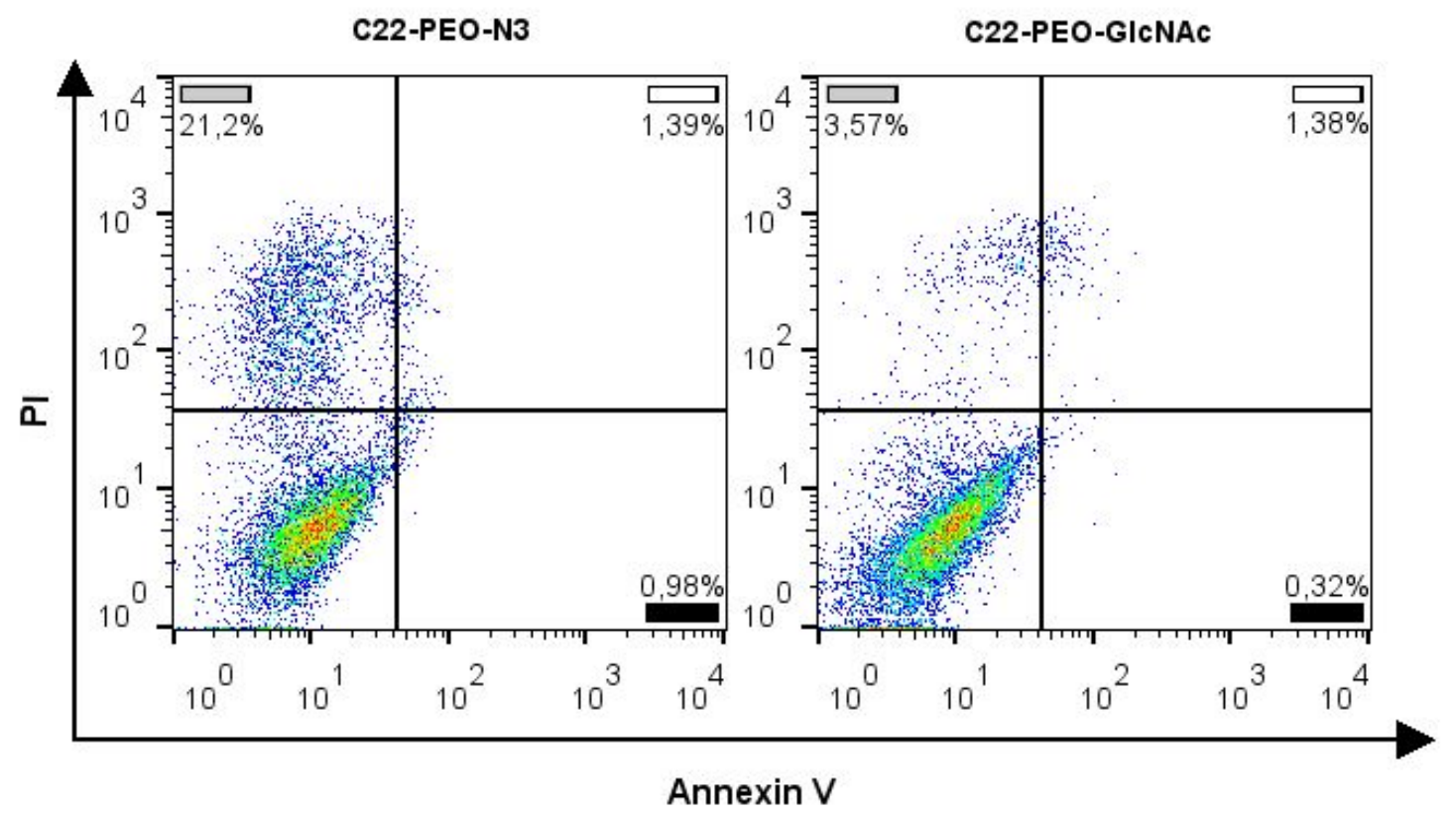

Figure S8. 2D dot-plots for the determination of live, apoptotic and necrotic cells exposed to $\mathrm{C}_{22} \mathrm{PEO}_{900}-\mathrm{N}_{3}$ and $\mathrm{C}_{22} \mathrm{PEO}_{900}$-GlcNAc nanoparticles at $c=100 \mu \mathrm{g} \cdot \mathrm{mL}^{-1}$ for $24 \mathrm{~h}$ 


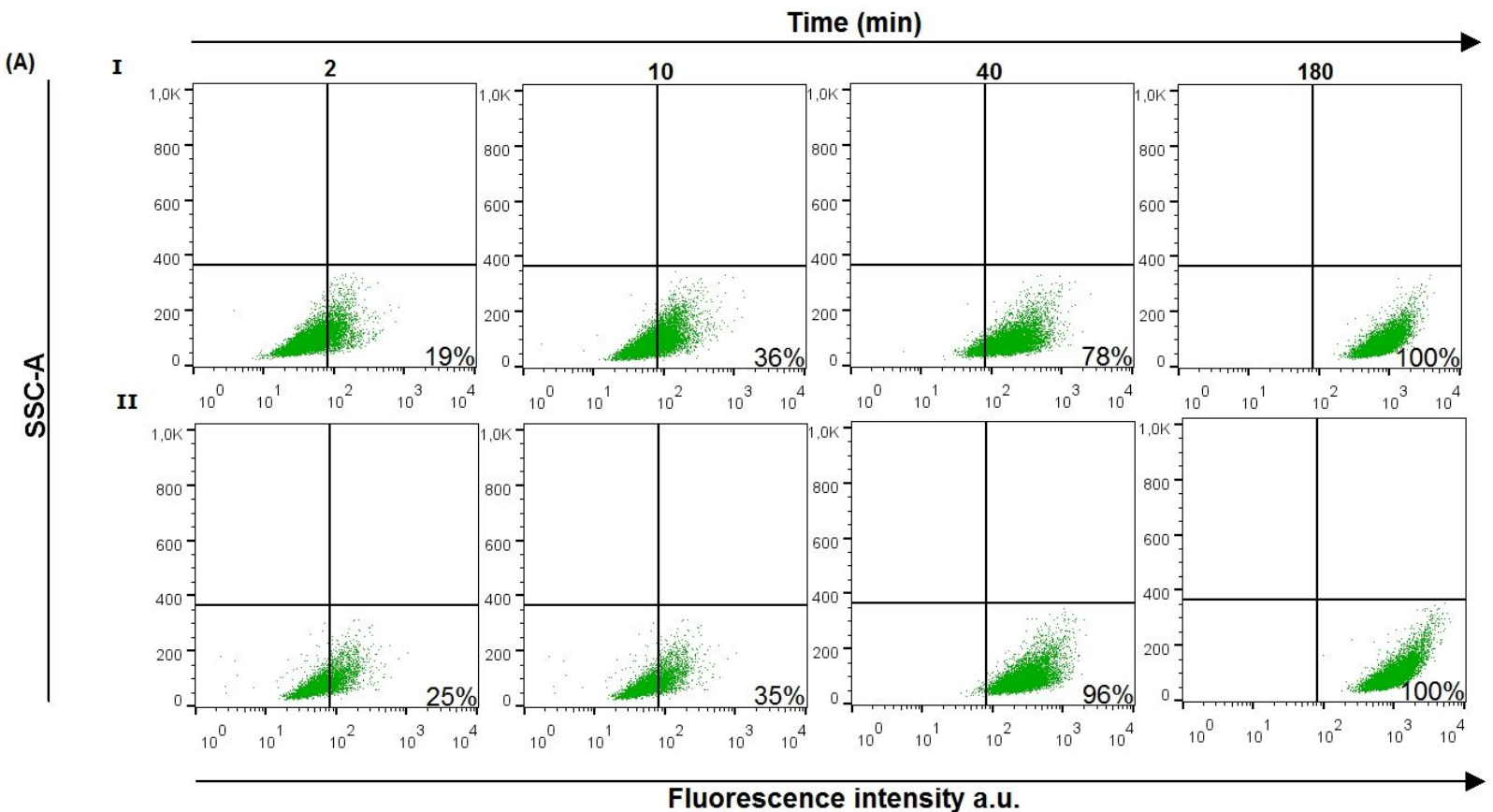

(B)

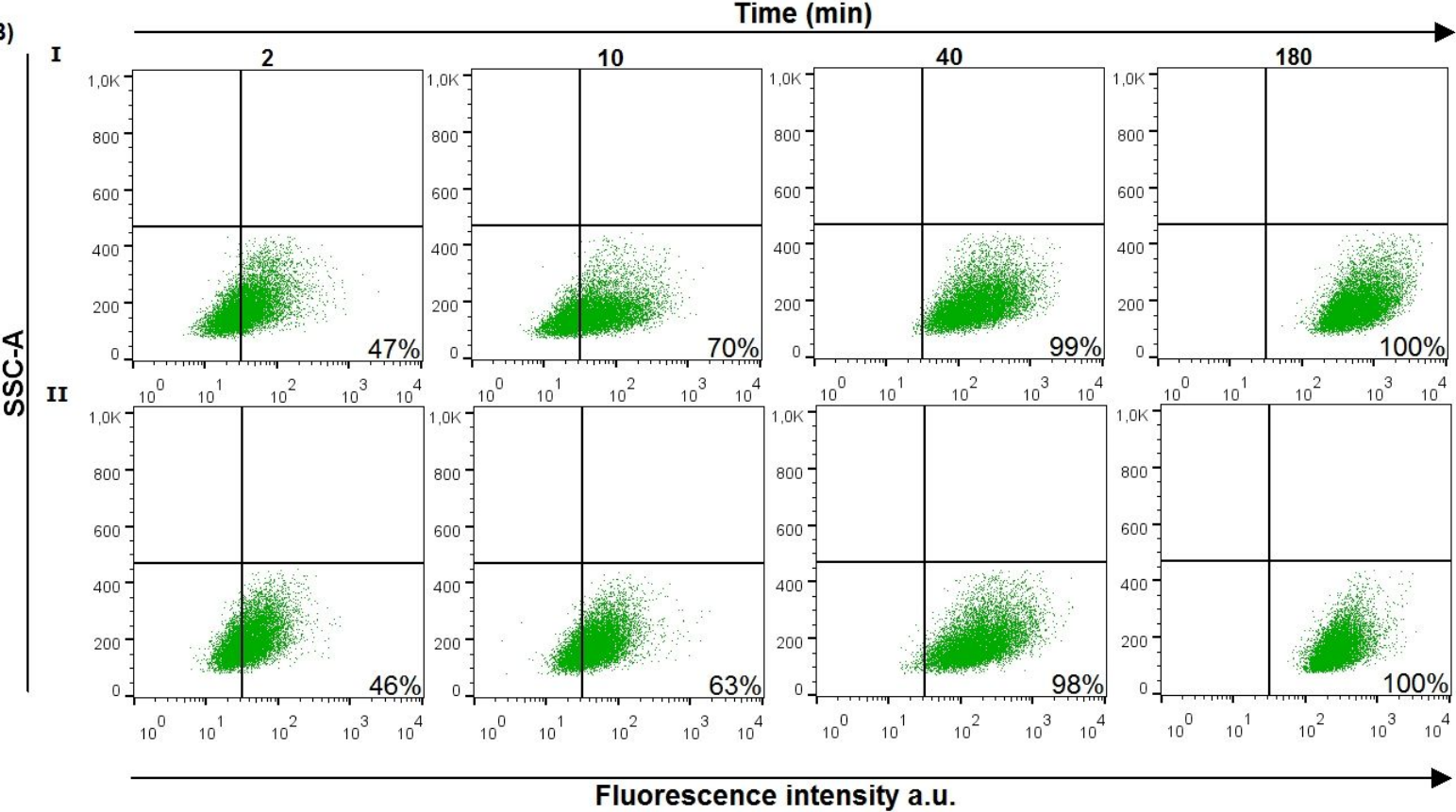

Figure S9. Flow cytometry dot plots portraying the evolution of cellular uptake in Telo$\mathrm{RF}$ (top) and HeLa (bottom) cells (I refers to $\mathrm{C}_{22} \mathrm{PEO}_{900}$-GlcNAc and II refers to $\left.\mathrm{C}_{22} \mathrm{PEO}_{900}-\mathrm{N}_{3}\right)$. 
(A)

\section{Cell autofluorescence}
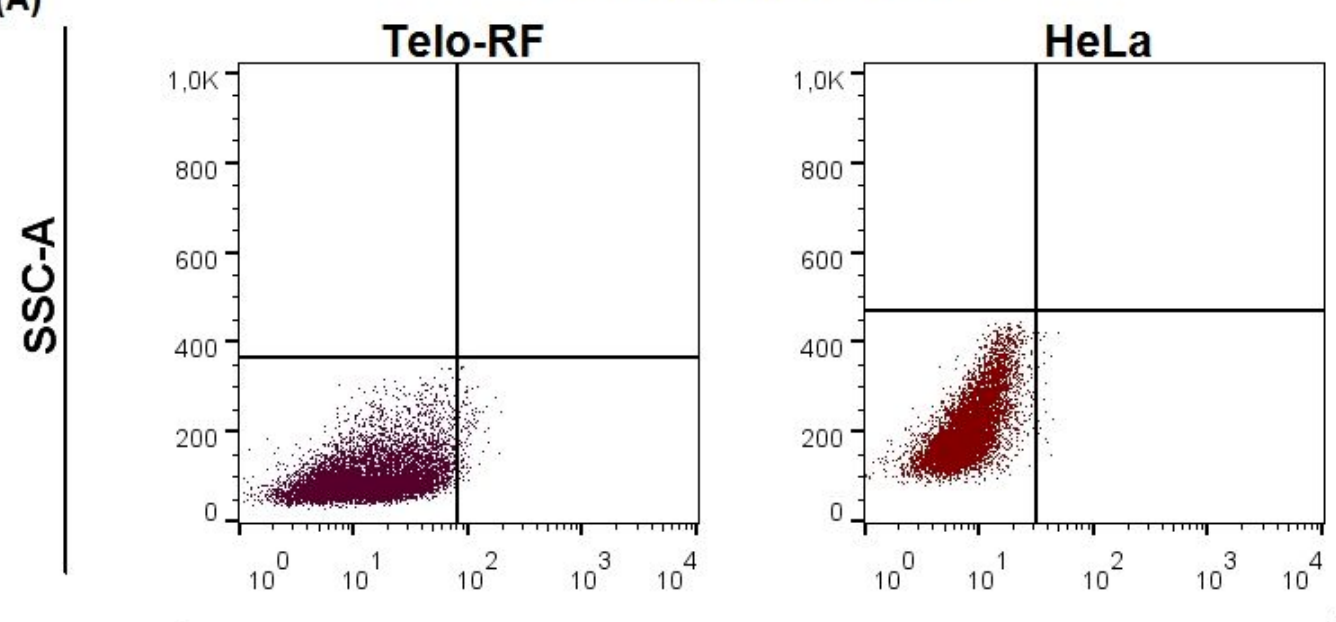

Fluorescence intensity a.u.

Figure S10. Flow cytometry dot plots portraying autofluoresence of Telo RF and HeLa cells used as the controls.

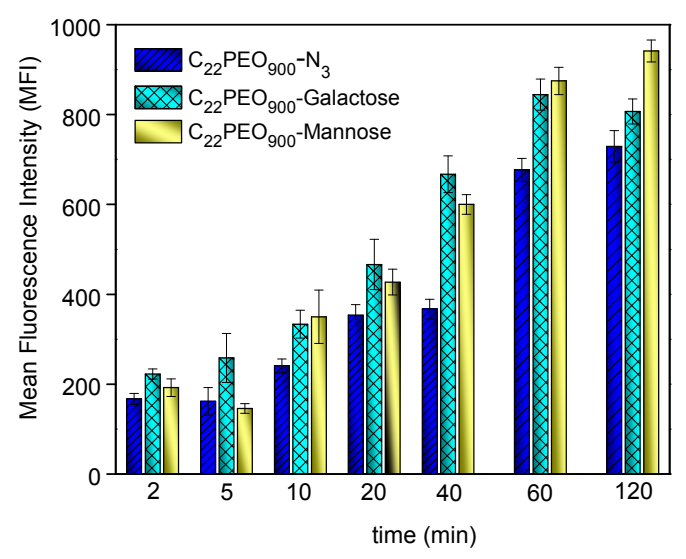

Figure S11. Evolution of cellular uptake for $\mathrm{C}_{22} \mathrm{PEO}_{900} \mathrm{~N}_{3}, \mathrm{C}_{22} \mathrm{PEO}_{900}$-Galactose and $\mathrm{C}_{22} \mathrm{PEO}_{900}$-Mannose nanoparticles incubated with HeLa cells (M.F.I. vs. time). 
Table S1. In-vitro stability of $\mathrm{C}_{22} \mathrm{PEO}_{900}-\mathrm{N}_{3}$ and $\mathrm{C}_{22} \mathrm{PEO}_{900}$-GlcNAc nanoparticles in PBS (pH 7.4) as a function of time.

\begin{tabular}{|c|c|c|c|c|}
\hline \multirow{2}{*}{ time (h) } & \multicolumn{2}{|c|}{$\mathrm{C}_{22} \mathrm{PEO}_{900}-\mathrm{N}_{3}$} & \multicolumn{2}{c|}{$\mathrm{C}_{22}$ PEO $_{900}$-GlcNAc } \\
\cline { 2 - 5 } & $\boldsymbol{R}_{\mathbf{H}}$ (nm) & Int. (kcps) & $\boldsymbol{R}_{\mathbf{H}}$ (nm) & Int. (kcps) \\
\hline 0 & 9.4 & 66.0 & 10.5 & 84.2 \\
\hline 24 & 9.8 & 72.0 & 10.3 & 82.8 \\
\hline 72 & 9.4 & 68.0 & 10.4 & 82.1 \\
\hline 168 & 9.6 & 71.0 & 10.7 & 83.0 \\
\hline
\end{tabular}

\section{Statistical Analysis}

2. Flow Cytometry Data

Table S2. Statistical analysis of the data reported in Figure 6: $\mathrm{C}_{22} \mathrm{PEO}_{900}-\mathrm{N}_{3}$ and $\mathrm{C}_{22} \mathrm{PEO}_{900}$-GlcNAc labelled nanoparticles uptaken by Telo-RF and HeLa cells as a function of the incubation time: "Yes" refers to statistically significant difference.

\begin{tabular}{|c|c|c|c|}
\hline \multirow[b]{2}{*}{ Evaluation } & \multirow[b]{2}{*}{ Time (min) } & \multicolumn{2}{|c|}{ Significant Difference } \\
\hline & & Telo-RF & $\mathrm{HeLa}$ \\
\hline $\begin{array}{c}\mathrm{C}_{22} \mathrm{PEO}_{900}-\mathrm{N}_{3} \text { compared to } \\
\mathrm{C}_{22} \mathrm{PEO}_{900}-\mathrm{GlcNAc}\end{array}$ & 2 & No & No \\
\hline $\begin{array}{c}\mathrm{C}_{22} \mathrm{PEO}_{900}-\mathrm{N}_{3} \text { compared to } \\
\mathrm{C}_{22} \mathrm{PEO}_{900}-\mathrm{GlcNAc}\end{array}$ & 5 & Yes & Yes \\
\hline $\begin{array}{c}\mathrm{C}_{22} \mathrm{PEO}_{900}-\mathrm{N}_{3} \text { compared to } \\
\mathrm{C}_{22} \mathrm{PEO}_{900} \text {-GlcNAc }\end{array}$ & 10 & No & Yes \\
\hline $\begin{array}{c}\mathrm{C}_{22} \mathrm{PEO}_{900}-\mathrm{N}_{3} \text { compared to } \\
\mathrm{C}_{22} \mathrm{PEO}_{900}-\mathrm{GlcNAc}\end{array}$ & 20 & Yes & Yes \\
\hline $\begin{array}{c}\mathrm{C}_{22} \mathrm{PEO}_{900}-\mathrm{N}_{3} \text { compared to } \\
\mathrm{C}_{22} \mathrm{PEO}_{900} \text {-GlcNAc }\end{array}$ & 40 & Yes & Yes \\
\hline $\begin{array}{c}\mathrm{C}_{22} \mathrm{PEO}_{900}-\mathrm{N}_{3} \text { compared to } \\
\mathrm{C}_{22} \mathrm{PEO}_{900} \text {-GlcNAc }\end{array}$ & 60 & No & Yes \\
\hline $\begin{array}{c}\mathrm{C}_{22} \mathrm{PEO}_{900}-\mathrm{N}_{3} \text { compared to } \\
\mathrm{C}_{22} \mathrm{PEO}_{900}-\mathrm{GlcNAc}\end{array}$ & 120 & Yes & Yes \\
\hline $\begin{array}{c}\mathrm{C}_{22} \mathrm{PEO}_{900}-\mathrm{N}_{3} \text { compared to } \\
\mathrm{C}_{22} \mathrm{PEO}_{900} \text {-GlcNAc }\end{array}$ & 180 & No & Yes \\
\hline
\end{tabular}


Tables S3-S4. Statistical analysis of the data reported in Figure S4: $\mathrm{C}_{22} \mathrm{PEO}_{900}-\mathrm{N}_{3}$, $\mathrm{C}_{22} \mathrm{PEO}_{900}$-Galocatose and $\mathrm{C}_{22} \mathrm{PEO}_{900}$-Mannose labelled nanoparticles uptaken by $\mathrm{HeLa}$ cells as a function of the incubation time: "Yes" refers to statistically significant difference.

Significant Difference

\begin{tabular}{|c|c|c|}
\hline Evaluation & Time (min) & HeLa \\
\hline $\begin{array}{c}\mathrm{C}_{22} \mathrm{PEO}_{900}-\mathrm{N}_{3} \text { compared to } \\
\mathrm{C}_{22} \mathrm{PEO}_{900} \text {-Mannose }\end{array}$ & 2 & No \\
\hline $\begin{array}{c}\mathrm{C}_{22} \mathrm{PEO}_{900}-\mathrm{N}_{3} \text { compared to } \\
\mathrm{C}_{22} \mathrm{PEO}_{900} \text {-Mannose }\end{array}$ & 5 & No \\
\hline $\begin{array}{c}\mathrm{C}_{22} \mathrm{PEO}_{900}-\mathrm{N}_{3} \text { compared to } \\
\mathrm{C}_{22} \mathrm{PEO}_{900} \text {-Mannose }\end{array}$ & 10 & Yes \\
\hline $\begin{array}{c}\mathrm{C}_{22} \mathrm{PEO}_{900}-\mathrm{N}_{3} \text { compared to } \\
\mathrm{C}_{22} \mathrm{PEO}_{900} \text {-Mannose }\end{array}$ & 20 & Yes \\
\hline $\begin{array}{c}\mathrm{C}_{22} \mathrm{PEO}_{900}-\mathrm{N}_{3} \text { compared to } \\
\mathrm{C}_{22} \mathrm{PEO}_{900} \text {-Mannose }\end{array}$ & 40 & Yes \\
\hline $\begin{array}{c}\mathrm{C}_{22} \mathrm{PEO}_{900}-\mathrm{N}_{3} \text { compared to } \\
\mathrm{C}_{22} \mathrm{PEO}_{900} \text {-Mannose }\end{array}$ & 60 & Yes \\
\hline $\mathrm{C}_{22} \mathrm{PEO}_{900}-\mathrm{N}_{3}$ compared to \\
$\mathrm{C}_{22} \mathrm{PEO}_{900}$-Mannose
\end{tabular}

Significant Difference

\begin{tabular}{|c|c|c|}
\hline Evaluation & Time (min) & HeLa \\
\hline $\begin{array}{c}\mathrm{C}_{22} \mathrm{PEO}_{900}-\mathrm{N}_{3} \text { compared to } \\
\mathrm{C}_{22} \mathrm{PEO}_{900} \text {-Galactose }\end{array}$ & 2 & Yes \\
\hline $\begin{array}{c}\mathrm{C}_{22} \mathrm{PEO}_{900}-\mathrm{N}_{3} \text { compared to } \\
\mathrm{C}_{22} \mathrm{PEO}_{900} \text {-Galactose }\end{array}$ & 5 & Yes \\
\hline $\begin{array}{c}\mathrm{C}_{22} \mathrm{PEO}_{900}-\mathrm{N}_{3} \text { compared to } \\
\mathrm{C}_{22} \mathrm{PEO}_{900} \text {-Galactose }\end{array}$ & 10 & Yes \\
\hline $\begin{array}{c}\mathrm{C}_{22} \mathrm{PEO}_{900}-\mathrm{N}_{3} \text { compared to } \\
\mathrm{C}_{22} \mathrm{PEO}_{900} \text {-Galactose }\end{array}$ & 20 & Yes \\
\hline $\begin{array}{c}\mathrm{C}_{22} \mathrm{PEO}_{900}-\mathrm{N}_{3} \text { compared to } \\
\mathrm{C}_{22} \mathrm{PEO}_{900} \text {-Galactose }\end{array}$ & 40 & Yes \\
\hline $\begin{array}{c}\mathrm{C}_{22} \mathrm{PEO}_{900}-\mathrm{N}_{3} \text { compared to } \\
\mathrm{C}_{22} \mathrm{PEO}_{900} \text {-Galactose }\end{array}$ & 60 & Yes \\
\hline $\mathrm{C}_{22} \mathrm{PEO}_{900}-\mathrm{N}_{3}$ compared to \\
$\mathrm{C}_{22} \mathrm{PEO}_{900}$-Galactose
\end{tabular}

